\title{
In Achilles Tendon Disorders, Will Sonoelastography Add to Grey-Scale Ultrasound? Using MRI as Gold Standard
}

\author{
Nour Mohamed Kandil ${ }^{1} \quad$ Maha Ahmed Abdelkarim ${ }^{1, \odot}$ \\ Aya Mohamed Hashem ${ }^{1, \odot}$ \\ ${ }^{1}$ Musculoskeletal Imaging Unit, Department of Radiology, Faculty of \\ Medicine, Cairo University, Cairo, Egypt
}

\author{
Nagui Mohamed Abdelwahab
}

\begin{abstract}
Address for correspondence Nour Mohamed Kandil, MD, Musculoskeletal Imaging Unit, Department of Radiology, Faculty of Medicine, Cairo University, Cairo, Egypt (e-mail: dr.n.kandil@hotmail.com).
\end{abstract}

\begin{abstract}
Keywords

- sonoelastography

- Achilles tendon

- grey scale ultrasound

$-M R I$
\end{abstract}

\section{Background}

The number of overuse tendon injuries has increased (30$50 \%$ of all sports-related injuries), including the Achilles tendon, as a common health problem in middle-aged active people. ${ }^{1}$ The imaging of the Achilles tendon can be done with several modalities; magnetic resonance imaging (MRI) and ultrasound (US) being the most commonly used of them. ${ }^{2}$

US is readily accessible and quick, with the possibility of interaction with the patient and the addition of power Doppler, which can help in examining the blood flow within and around the tendon. Moreover, US elastography can evaluate the mechanical properties of tendons (soft vs. hard). ${ }^{1,2}$
US elastography is a relatively new, real-time imaging technique which depends on tissue compression. The most commonly used elastographic technique is sonoelastography (SEL), also described as strain elastography and freehand compression elastography. The technique is based on manual compression of the tissue, which is usually applied by the hand-held US transducer. ${ }^{1,2}$

We aimed to assess the feasibility of using SEL in cases with Achilles tendon disorders, and to verify its findings against US and MRI. We also aimed to describe the different elastographic patterns and correlate the results to MRI as a gold standard. published online July 27,2021
DOI https://doi.org/

10.1055/s-0041-1734224 ISSN 0971-3026

\section{(C) 2021. Indian Radiological Association.}

This is an open access article published by Thieme under the terms of the Creative Commons Attribution-NonDerivative-NonCommercial-License, permitting copying and reproduction so long as the original work is given appropriate credit. Contents may not be used for commercial purposes, or adapted, remixed, transformed or built upon. (https://creativecommons.org/licenses/by-nc-nd/4.0/).

Thieme Medical and Scientific Publishers Private Ltd. A-12, Second Floor, Sector -2, NOIDA -201301, India 


\section{Methods}

The current study had been approved by Kasr El-Aini Hospital, Research and Ethical committee.

This study was performed on 60 symptomatic patients (51 males and nine females) referred to us from either the orthopaedic or rheumatology outpatient clinics, their age ranged from 33 to 59 years (mean age 45 ).

All patients were complaining of pain related to the Achilles tendon (the right in 53 patients and the left in seven patients). The duration of symptoms ranged from 2 years to 1 month with a mean duration of 9.3 months. Three patients had a history of Achilles tendon operation. Whenever bilateral complaint was present, the more symptomatizing side was included in our study.

Six patients were suffering from rheumatoid arthritis, 10 were diabetics, and five were hypertensive while the rest of the patients had no concomitant disease.

All patients were subjected to relevant history taking and local examination, Achilles tendon US, and elastography in the same setting and ankle MRI examination.

\section{Ultrasound Examination}

\section{Technique of the Examination}

The examination of all patients included B-mode scanning and SEL in the same setting using the same US system (Aplio $\mathrm{mx}$, Toshiba Medical Systems, Japan) and the same linear transducer (7-12 Mhz).

Each Achilles tendon was examined by B-mode US with the patient in the prone position and the foot hanging over the edge of the examination bed in a relaxed position to avoid tendon stress, and copious amounts of US gel was used. Examination included the full length of the tendon in both longitudinal and transverse planes.

Subsequently SEL was performed by applying moderate repetitive compression with the hand-held transducer over the area of interest with the patient in the same position. Sonoelastographic images of the tendons were obtained in the longitudinal plane after a few seconds of processing (off-line procedure). Longitudinal views were easier to acquire and more reproducible than transverse views. The elastogram appeared within a rectangular region of interest (ROI) as a translucent color-coded image superimposed on the B-mode image. The color code indicated the relative stiffness of the tissues within the ROI and ranged from red (hard) to blue (soft) with yellow and green colors representing medium stiffness and the same color scale was used in all patients. This color code was designed by the US manufacturer and it should be noted that it is different from the most commonly used color code. At least three compression-decompression cycles were applied to improve the signal-to-noise ratio in the generated image. At least three SEL images were obtained of each tendon half; finally, the most representative image of the three was chosen for image interpretation.

\section{Ultrasound Image Analysis}

Ultrasound Interpretation

Each tendon was evaluated for: anteroposterior thickness measured 2 to $4 \mathrm{~cm}$ proximal to the calcaneus, fibrillar pattern, echogenicity, intact parallel margins, and insertion. Presence of bursae, calcifications, interstitial or complete tear was also considered.

\section{Elastographic Image Interpretation}

The elastographic images were qualitatively reviewed by visual inspection to determine the elastographic pattern represented by the color map: hard tendon fibers (red and yellow), mild softening (green), and marked softening (blue). Every tendon was divided into proximal, middle, and distal thirds, with each third divided into superficial and deep fibers and correlation to the corresponding areas on US was done. Due to heterogeneity of the elastograms in the examined tendons the following grading system was developed:

Grade 1: Area of marked softening (blue) about $0-25 \%$ of the tendon.

Grade 2: Area of marked softening (blue) about 25-50\% of the tendon.

Grade 3: Area of marked softening (blue) more than $50 \%$ of the tendon.

In our results, we considered each Achilles tendon as grade 1 when all thirds were characterized as grade 1 , as grade 2 when at least one-third showed alterations of grade 2, and as grade 3 when at least one-third showed alteration of grade 3, i.e., the tendon was given the grade of the most advanced changes seen within any of its segments. This grading system was used for correlation to MRI.

\section{MRI Examination}

\section{Technique of the Examination}

All MR studies were performed on a 1.5-T MR imaging unit. Patients were placed in the supine position and a dedicated ankle coil was used. Imaging sequences included at least sagittal T2 and STIR-weighted images as well as axial T1 and T2-weighted sequences covering the whole length of the Achilles tendon from the calcaneal insertion to the myotendinous junction .Other sequences and planes were added whenever it was required.

\section{MRI Image Analysis}

MRI interpretation was done regarding tendon thickness, insertional irregularities, and abnormal signals (degeneration, calcifications, and tears). The images were also graded from 1 to 3 :

Grade 1: represented a normal tendon.

Grade 2: represented thickened tendon with homogeneous signal.

Grade 3: described intratendinous signal intensity change (with or without tendon thickening).

\section{Statistics}

Data were statistically described in terms of mean \pm standard deviation ( $\pm \mathrm{SD}$ ), and range, or frequencies (number of cases) and percentages when appropriate. Agreement between elastography and MRI grade was done using kappa statistic. $p$-Values less than 0.05 was considered statistically significant. All statistical calculations were done using computer program SPSS (Statistical Package for the Social Science; SPSS Inc., Chicago, Illinois, United States) version 15 for Microsoft Windows. 


\section{Results}

This study was performed on 60 symptomatic patients (51 males and 9 females) referred to us from either the orthopaedic or rheumatology outpatient clinics, their age ranged from 33 to 59 years (mean age 45).

All patients were complaining of pain related to the Achilles tendon (the right in 53 patients and the left in seven patients). The duration of symptoms ranged from 2 years to 1 month with mean duration of 9.3 months. Three patients had a history of Achilles tendon operation. Whenever bilateral complaint was present, the more symptomatizing side was included in our study.

Six patients were suffering from rheumatoid arthritis, 10 were diabetics, and five were hypertensive while the rest of the patients had no concomitant disease. By US examination the following pathological entities were recognized (summarized in - Table $\mathbf{1}$ ).

\section{Tendon Thickening}

The tendon is considered thickened when the anteroposterior diameter is more than $6.5 \mathrm{~mm}$.Our study included 48 thickened tendons while overall thickness ranges from 6.1 to $9.7 \mathrm{~mm}$ (mean thickness $7.9 \mathrm{~mm}$, and SD = 1.12) in the study subjects (-Figs. 1, 13, 15, and 16).

\section{Hypoechogenicity}

The tendon is noted to have an inhomogeneous texture with focal areas of hypoechogenicity. Our study included 36 cases in this group (-Figs. 1, 7, 10, 13, and 16, 17).

\section{Interrupted Pattern}

Loss of the normal fibrillar pattern in any of the tendon parts was observed in 54 cases in our study (-Figs. 1, 7, 10, 13, and 16).

\section{Partial Tear}

Ultrasound detected partial interruption of tendon fibers as a linear hypoechoic band which was either traversing the tendon fibers or splitting them. Our study included 12 cases in this group (-Figs. 1, 10, and 13).

Table 1 Frequency of ultrasonographically recognized Achilles tendon pathologies among the study subjects

\begin{tabular}{|l|l|l|}
\hline Pathological feature & $\begin{array}{l}\text { Number of } \\
\text { cases }\end{array}$ & Percentage \\
\hline $\begin{array}{l}\text { Increased anteroposterior } \\
\text { thickness }\end{array}$ & 48 & $80 \%$ \\
\hline $\begin{array}{l}\text { Interruption of fibrillary } \\
\text { pattern }\end{array}$ & 54 & $90 \%$ \\
\hline $\begin{array}{l}\text { Focal areas of } \\
\text { hypoechogenicity }\end{array}$ & 36 & $60 \%$ \\
\hline $\begin{array}{l}\text { Interstitial tear (longitudinal } \\
\text { fiber splitting) }\end{array}$ & 12 & $20 \%$ \\
\hline Insertional irregularity & 42 & $70 \%$ \\
\hline Insertional calcifications & 18 & $30 \%$ \\
\hline Bursitis & 12 & $20 \%$ \\
\hline
\end{tabular}

\section{Enthesopathy}

Enthesopathy was diagnosed with irregularity of the insertion site of the calcaneus with or without calcification of the tendinous insertion. Insertional irregularities were found in 32 cases with associated calcification in 18 cases (-Figs. 4, 6, 7, 10, and 16).
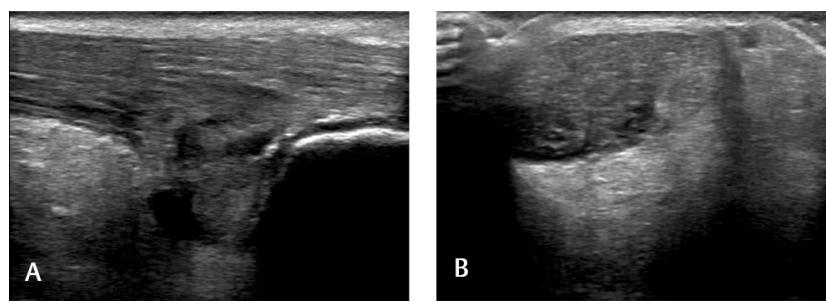

Fig. 1 Ultrasound of the right Achilles tendon (case n. 1), (A) longitudinal view, showing loss of fibrillar pattern, thickening, hypoechoic foci, and bursitis. (B) Transverse view showing concave anterior surface of the tendon with hypoechoic deep fibers.
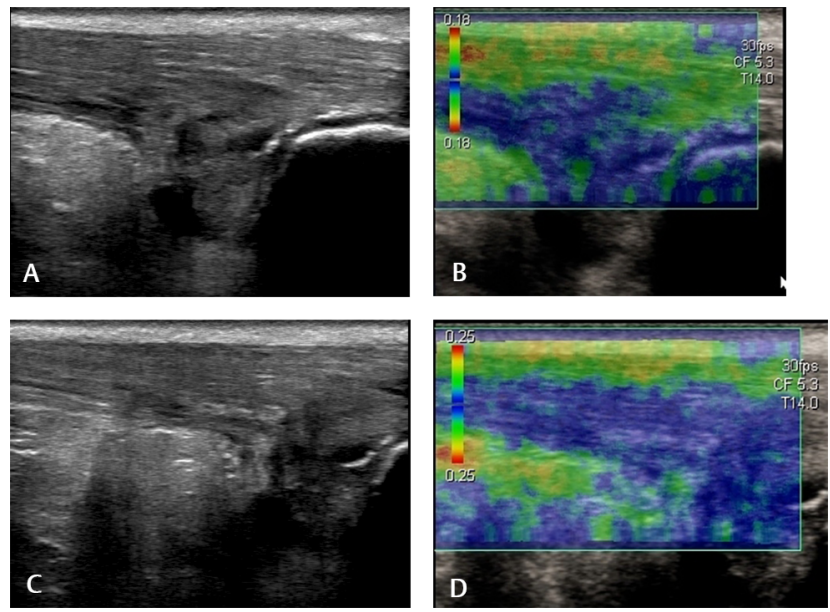

Fig. 2 Elastographic color map of the Achilles tendon (case n. 1): (A) longitudinal view ultrasound with (B) corresponding elastograms of the distal third. (C) Ultrasound of a more proximal part with overlapping area with the previous image, and (D) the corresponding elastograms. All show marked softening (blue) of the deep fibers and mild softening (green) of the superficial fibers.
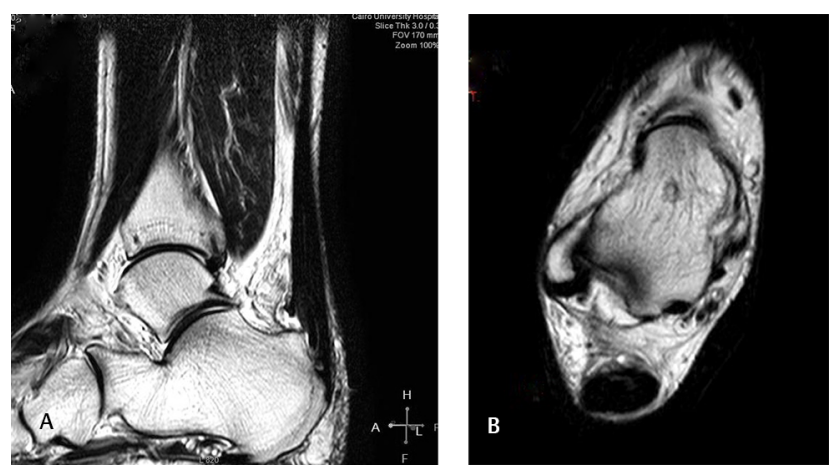

Fig. 3 MRI of the ankle (case no.1), (A) sagittal T2 WI, and (B) axial $\mathrm{T} 2 \mathrm{WI}$, showing thickened Achilles tendon with areas of intermediate signal, partial tear, and bursitis. MRI, magnetic resonance imaging. 


\section{Bursitis}

Retrocalcaneal bursitis represented by a bursal sac measuring more than $2 \mathrm{~mm}$ in anteroposterior diameter, which was found in 12 cases (-Fig. $\mathbf{1}$ ).

For interpretation of sonoelastography (SEL) images, each tendon was divided longitudinally into deep and superficial fibers.
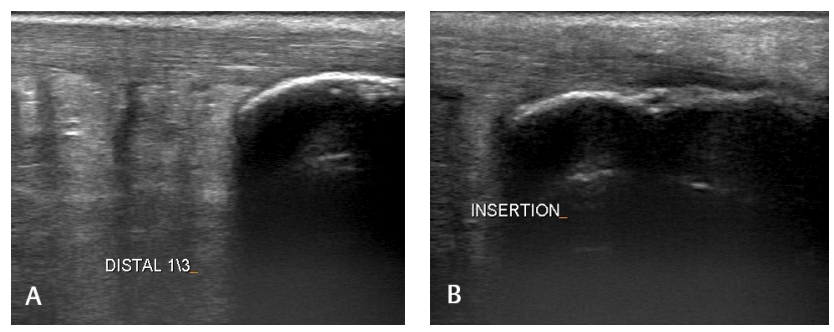

Fig. 4 Ultrasound of the Achilles tendon (case n. 2). (A) Normal distal third, (B) calcaneal insertion showing mild irregularity.
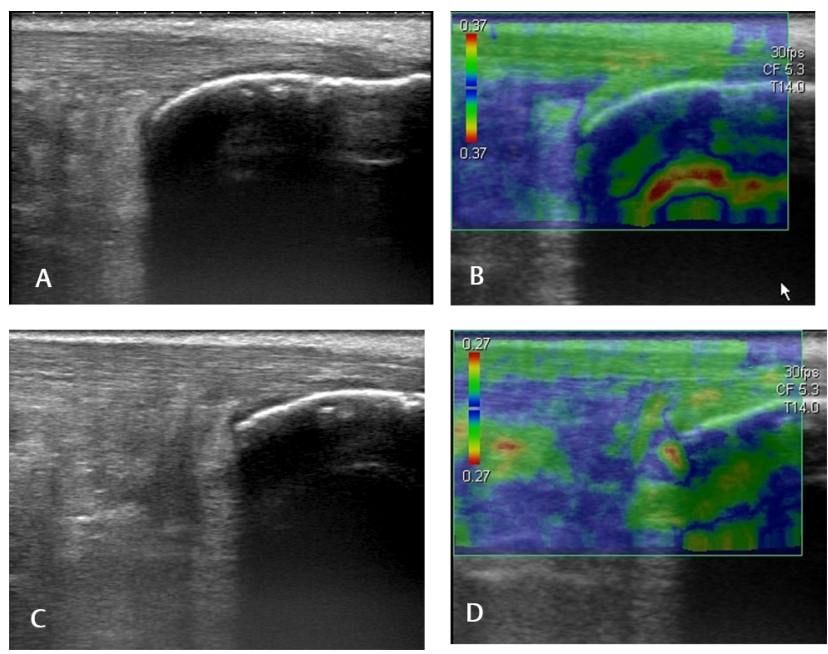

Fig. 5 Elastography of the Achilles tendon (case n. 2): (A) longitudinal view ultrasound of the distal third with (B) corresponding elastograms. (C) Ultrasound of a more proximal part with overlapping area with the previous image, and (D) the corresponding elastograms. All show the tendon fairly homogenous green in color representing mild softening.
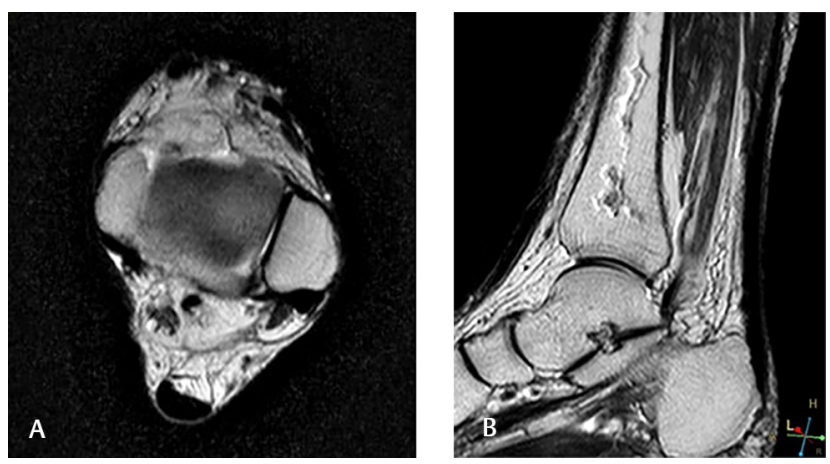

Fig. 6 MRI of the ankle (case n. 2). (A) Axial T2WI and (B) sagittal T2WI showing average tendon thickness with mild insertional irregularities. Bone infarction was noted in the lower tibia. MRI, magnetic resonance imaging
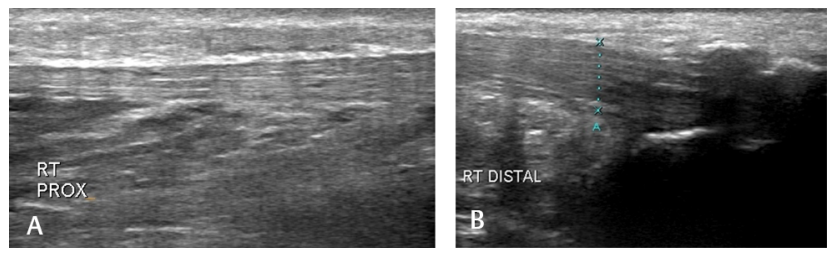

Fig. 7 Ultrasound of the Achilles tendon (case n. 3). (A) Proximal part with normal appearance, and (B) distal part showing hypoechoic area, calcification, and partial loss of fibrillar pattern.
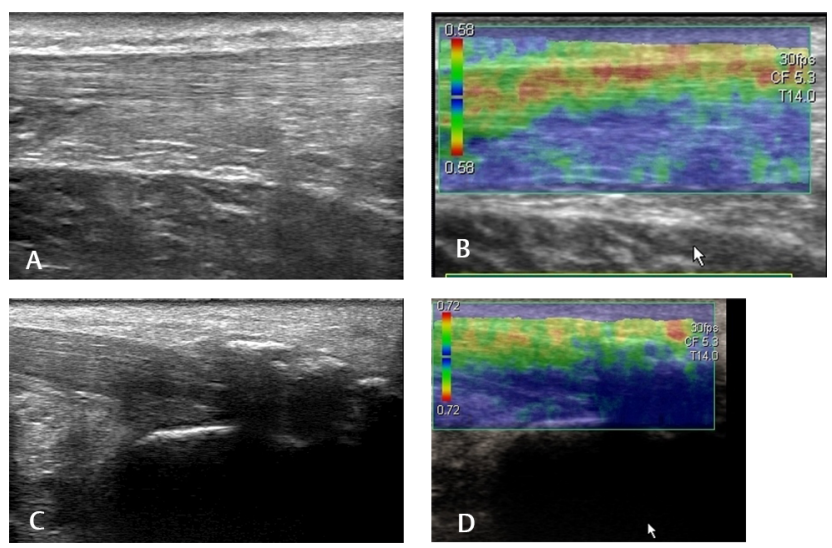

Fig. 8 Elastography of the Achilles tendon (case n. 3). (A) Longitudinal view ultrasound of the proximal part showed normal fibrillar pattern, (B) the corresponding elastograms appeared predominantly red/yellow denoting hard structure, (C) ultrasound of the distal part of the tendon showing calcifications and hypoechoic areas, and (D) the corresponding elastogram showed blue color (marked softening) of the deep fibers and green color (mild softening) of the superficial fibers.
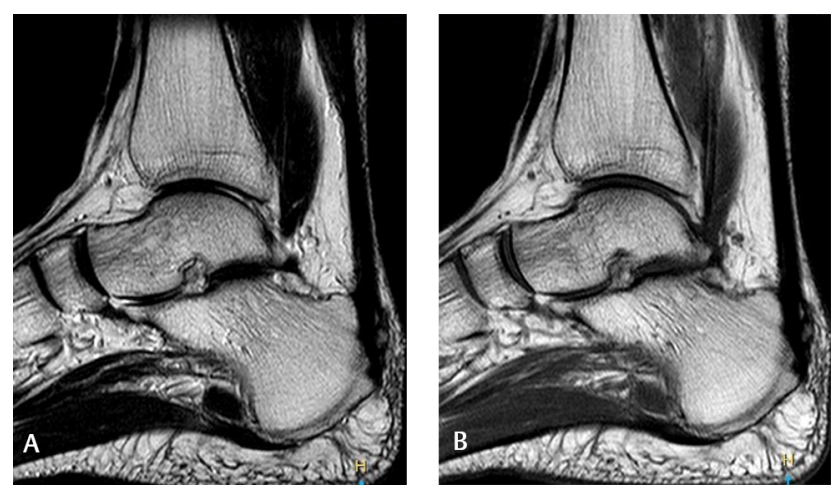

Fig. 9 MRI of the ankle (case n. 3). (A) Sagittal T2WI and (B) sagittal T1WI, both showed insertional thickening of the Achilles tendon, tendon ossification, and insertional irregularities. MRI, magnetic resonance imaging.

All 60 tendons showed variable degrees of softening, and all were heterogeneous in their elastographic color code so our interpretation depended on the predominant color. As illustrated in - Table 2, the deep fibers appeared predominantly blue (marked softening) in 42 tendons (70\%) (- Figs. 2, $\mathbf{8 , 1 1}$, and 14), while appeared predominantly green (mild softening) in 18 tendons (30\%) ( - Fig. 5). The superficial fibers appeared predominantly red/yellow (normal hard structure) in six tendons (10\%), appeared predominantly green (mild 

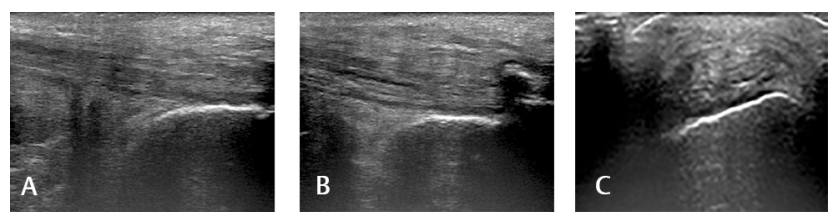

Fig. 10 Ultrasound of the Achilles tendon (case n. 4). (A) Longitudinal view of the distal third showing disrupted pattern and in (B) insertional irregularities and calcifications, with an interstitial tear seen splitting the fibers. (C) Transverse view showing thickened tendon with peritendinous fluid.
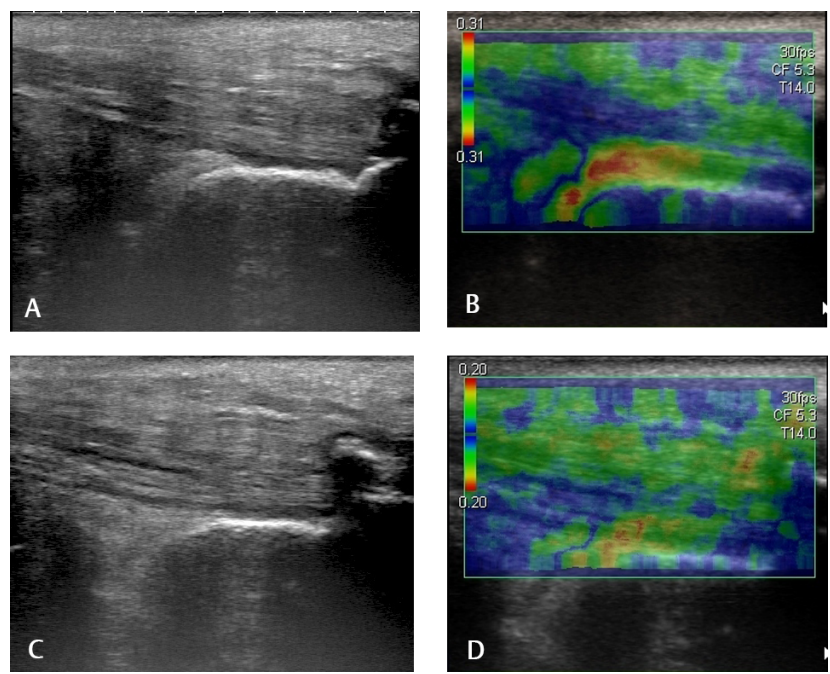

Fig. 11 Elastography of the Achilles tendon (case n. 4). (A), (C) ultrasound of the distal part of the tendon and (B), (D) the corresponding elastograms, respectively, showing heterogenous colors.
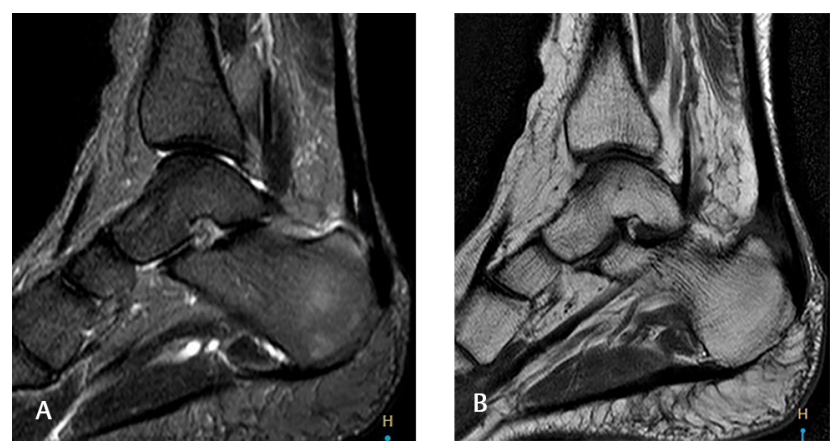

Fig. 12 MRI of the ankle (case n. 4). (A) Sagittal STIR WI and (B) sagittal T1WI showing tendon thickening, degenerative changes, and interstitial tear. MRI, magnetic resonance imaging.
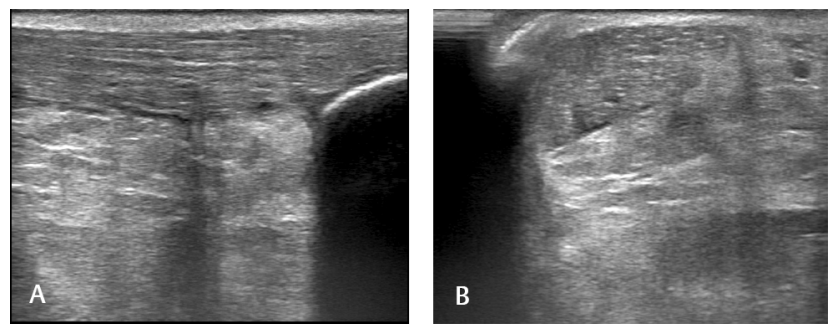

Fig. 13 Ultrasound of the Achilles tendon (case n. 5). (A) Longitudinal view and (B) transverse view, showing thickened tendon, generally hypoechoic with preserved fibrillar pattern. The anterior fibers showed a small linear interstitial tear.
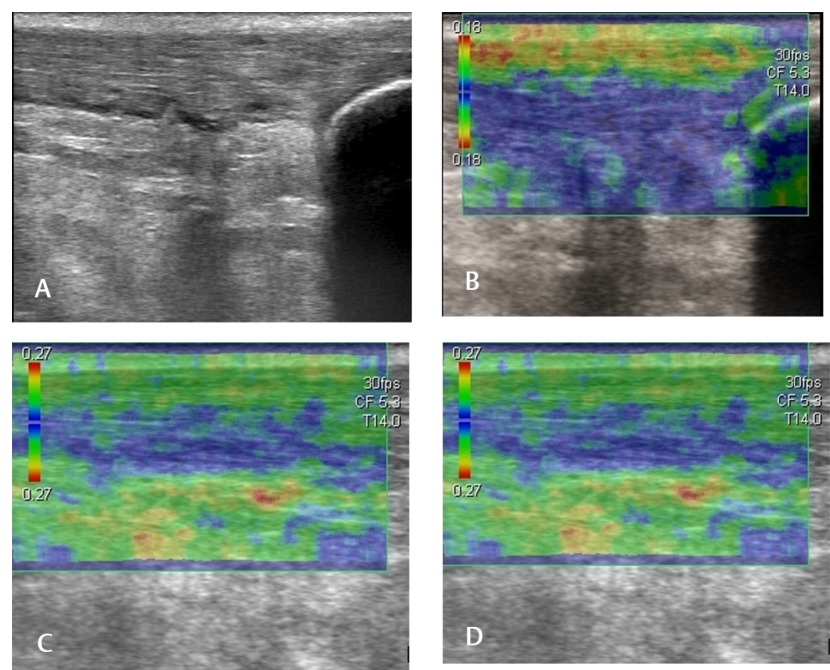

Fig. 14 Elastography of the Achilles tendon (case n. 5). (A) Ultrasound of the distal part of the tendon and (B) the corresponding elastogram with blue anterior fibers (marked softening) and red/yellow superficial fibers (hard). (C) Ultrasound of the proximal part of the tendon, (D) the corresponding elastogram with blue deep fibers (marked softening) and green posterior fibers (mild softening).
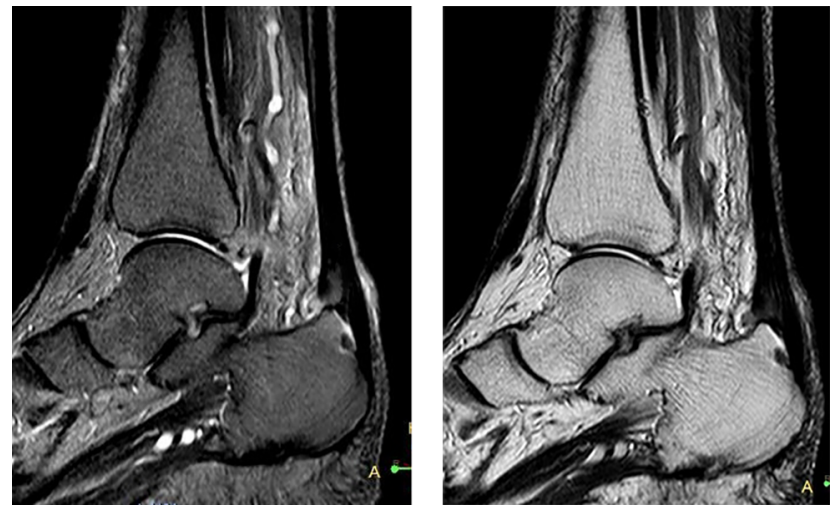

Fig. 15 MRI of the ankle (case n. 5). (A) Sagittal STIR WI and (B) sagittal T2WI showing thickened Achilles tendon with an area of abnormal intermediate signal affecting the anterior fibers, denoting tendon degeneration. MRI, magnetic resonance imaging.
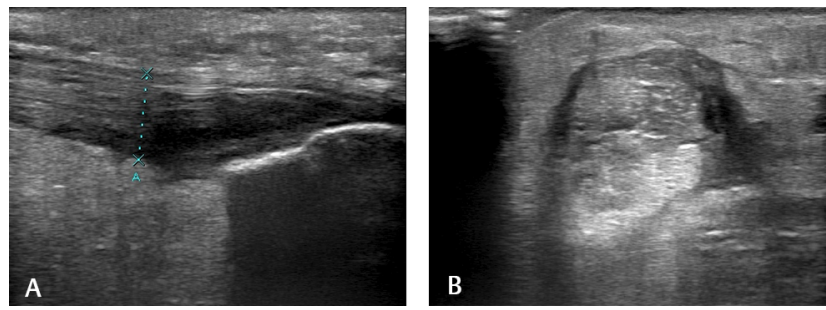

Fig. 16 Ultrasound of the Achilles tendon (case n. 8). (A) Longitudinal view of the distal third of the tendon showing a focal hypoechoic area, distance $A=7.3 \mathrm{~mm}$, (B) transverse view of the tendon showing the peritendinous fluid.

softening) in 48 tendons (80\%) (-Figs. $\mathbf{2 , 5 , 8}$, and $\mathbf{1 4}$ ), and appeared predominantly blue in six tendons (10\%) (-Fig. 11).

Matching of each elastographic color to the underlying B-mode image in each of the 120 tendon halves was done to test the ability of each elastographic color to spot an underlying ultrasound change. This is illustrated in - Table 3. 

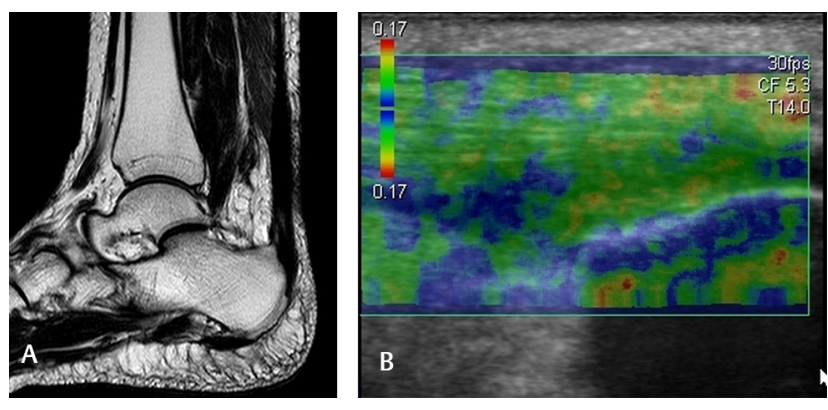

Fig. 17 Elastography of the Achilles tendon (case n. 8). (A) Ultrasound of the distal part of the tendon, and (B) the corresponding elastogram showing predominance of the green color (mild softening) with only a small area of blue color (marked softening). Here the area of ultrasound hypoechogenicity was found to be of mild softening.
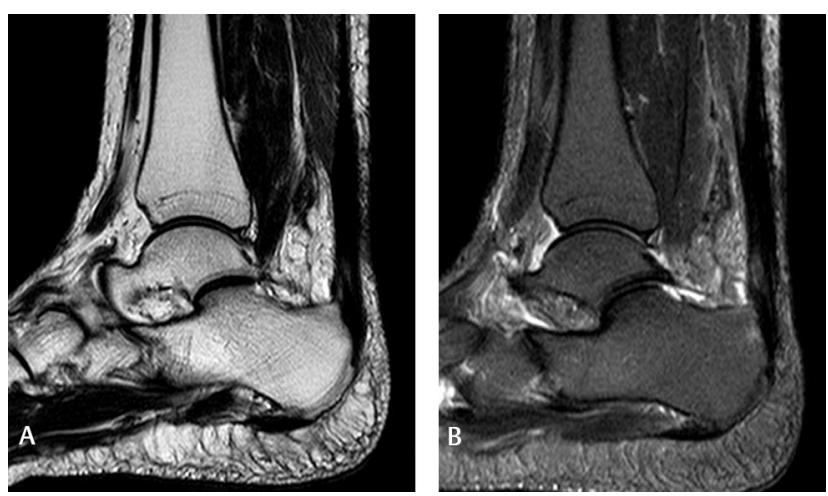

Fig. 18 MRI of the ankle (case n. 8). (A) Sagittal T2WI and (B) sagittal STIR WI, showing mildly thickened Achilles tendon with area of abnormal signal intensity within its distal part denoting tendon degeneration. MRI, magnetic resonance imaging.

Based on the previous analysis, considering US as our diagnostic reference:

Elastographic detection of softening (blue and green colors) has $100 \%$ sensitivity in detecting abnormalities that were detected by B-mode ultrasound with $16.6 \%$ specificity, positive predictive value of $73 \%$, and negative predictive value of $100 \%$.

The elastographic blue color representing marked softening appears to correspond more frequently with marked ultrasound changes (focal hypoechoic areas and interstitial tears) with sensitivity of $50 \%$ and specificity of $83.3 \%$ in detection of any of the total ultrasound changes. On the other hand, the green color representing mild softening corresponds more frequently with mild ultrasonographic changes (thickening, fibrillar pattern interruption, normal/minimal findings) with sensitivity of $50 \%$ and specificity of $33.3 \%$ in detecting any of the total ultrasound changes, see - Table 4.

For correlation to MRI, the studied tendons were categorized into three grades regarding the elastographic map, we used the blue color percent area of the tendon elastogram (representing marked softening) as the categorizing factor.

Grade 1: Area of marked softening (blue) about $0-25 \%$ of the tendon (-Fig. 5).

Grade 2: Area of marked softening (blue) about $25-50 \%$ of the tendon (-Fig. 2).

Grade 3: Area of marked softening (blue) more than 50\% of the tendon (-Fig. 14).

In our results, as shown in $\mathbf{- T a b l e ~} \mathbf{5}$, we considered each Achilles tendon as grade 1 when all thirds were characterized as grade 1 (in 18 tendons-30\%), as grade 2 when at least one-third showed alterations of grade 2 (in 30 tendons $-50 \%$ ), and as grade 3 when at least one-third showed alteration of grade 3 (in 12 tendons-20\%).

On MRI examination: several abnormalities were appreciated as demonstrated in - Table 6 .

Both MRI and ultrasound detected all cases of bursitis (12 cases), increased tendon thickness (48 cases), and interstitial tears (12 cases). Ultrasound detected 42 cases with insertional irregularities which was reliably noted in 24 cases by MRI. In one case which showed calcification by ultrasound examination, MRI revealed intratendinous signal intensity comparable to bone marrow in all pulse sequences. It was diagnosed as tendon ossification (-Fig. 9).

Table 2 Frequency of each elastographic pattern in the examined tendon fibers

\begin{tabular}{|l|l|l|l|}
\hline \multirow{2}{*}{ Fibers } & \multicolumn{3}{|c|}{ Elastographic color } \\
\cline { 2 - 4 } & Blue & Green & Red \\
\hline Deep fibers & 42 & 18 & - \\
\cline { 2 - 4 } & $70 \%$ & $30 \%$ & 6 \\
\hline Superficial fibers & 48 & 6 & $10 \%$ \\
\cline { 2 - 5 } & $80 \%$ & $10 \%$ & $10 \%$ \\
\hline
\end{tabular}

Table 3 The frequency of different ultrasound features with their corresponding elastographic color

\begin{tabular}{|c|c|c|c|c|}
\hline \multirow[t]{2}{*}{ US feature } & \multicolumn{3}{|c|}{ Elastographic color } & \multirow{2}{*}{$\begin{array}{l}\text { Total } \mathrm{n} \text {. detected } \\
\text { by US }\end{array}$} \\
\hline & Blue & Green & Red & \\
\hline Generalized hypoechogenicity/disrupted pattern & 6 & 36 & - & 42 \\
\hline Focal hypoechoic areas & 24 & 6 & - & 30 \\
\hline Interstitial tear & 12 & - & - & 12 \\
\hline Normal fibers/minimal findings & 6 & 24 & 6 & 36 \\
\hline
\end{tabular}


Table 4 Sensitivity and specificity of each elastographic pattern in spotting of the sonographically detected abnormalities

\begin{tabular}{|l|l|l|}
\hline $\begin{array}{l}\text { Elastographic } \\
\text { color }\end{array}$ & Specificity (\%) & Sensitivity(\%) \\
\hline Blue & $83 \%$ & $50 \%$ \\
\hline Green & $33 \%$ & $50 \%$ \\
\hline Both colors & $17 \%$ & $100 \%$ \\
\hline
\end{tabular}

Table 5 Distribution of the Achilles tendon elastographic grades in the study group

\begin{tabular}{|l|l|l|}
\hline $\begin{array}{l}\text { Elastographic } \\
\text { tendons grade }\end{array}$ & $\begin{array}{l}\text { Number of } \\
\text { tendons }\end{array}$ & Percentage (\%) \\
\hline Grade 1 & 18 & $30 \%$ \\
\hline Grade 2 & 30 & $50 \%$ \\
\hline Grade 3 & 12 & $20 \%$ \\
\hline
\end{tabular}

Table 6 Achilles tendon abnormalities detected by MRI

\begin{tabular}{|l|l|l|}
\hline MRI abnormality & N. of cases & Percentage (\%) \\
\hline $\begin{array}{l}\text { Increased tendon } \\
\text { thickness }\end{array}$ & 48 & $80 \%$ \\
\hline $\begin{array}{l}\text { Insertional } \\
\text { irregularity }\end{array}$ & 24 & $40 \%$ \\
\hline $\begin{array}{l}\text { Abnormal interme- } \\
\text { diate signal }\end{array}$ & 36 & $60 \%$ \\
\hline Interstitial tear & 12 & $20 \%$ \\
\hline Bursitis & 12 & $20 \%$ \\
\hline Haglund deformity & 24 & $40 \%$ \\
\hline
\end{tabular}

Abbreviation: MRI, magnetic resonance imaging.
Regarding MRI grades:

Grade 1 was found in 12 tendons (20\%) (-Fig. 3), grade 2 was also found in 12 tendons (20\%) ( - Fig. 9), while grade 3 was found in 36 tendons (60\%) ( - Fig. 12). This is illustrated in - Table 7.

Agreement between elastography and MRI:

Cross tabulation of MRI grades and elastographic grades is shown in - Table 8.

7. Considering MRI as the gold standard, we blotted each of the elastographic grades against its MRI equivalent. We must note that MRI and elastography grades are not reflecting the exact pathology but rather a rough guide to the severity of tendon affection.

$7 \%$ of grade I elastographic tendons were normal MRI tendon (Grade I), while 33.3\% of them were actually diseased tendons according to MRI (grade III)

All of grade II elastographic tendons were diseased tendons according to MRI, with $40 \%$ of them corresponding to MRI grade II and 60\% to MRI grade III.

Lastly, all grade III elastographic tendons were diseased tendons, with $100 \%$ of them corresponding to grade III of MRI.

Table 7 Distribution of the MRI grades of the Achilles tendons in the study group

\begin{tabular}{|l|l|l|}
\hline $\begin{array}{l}\text { MRI tendons } \\
\text { grade }\end{array}$ & $\begin{array}{l}\text { Number of } \\
\text { tendons }\end{array}$ & Percentage (\%) \\
\hline Grade 1 & 12 & $20 \%$ \\
\hline Grade 2 & 12 & $20 \%$ \\
\hline Grade 3 & 36 & $60 \%$ \\
\hline
\end{tabular}

Abbreviation: MRI, magnetic resonance imaging.

Table 8 Cross tabulation of MRI grades and elastographic grades in the study group

\begin{tabular}{|c|c|c|c|c|c|c|}
\hline & & & \multicolumn{3}{|c|}{ MRI grade } & \multirow[t]{2}{*}{ Total } \\
\hline & & & 1 & 2 & 3 & \\
\hline \multirow{12}{*}{$\begin{array}{l}\text { Elastography } \\
\text { grade } \\
\text { Elastography } \\
\text { grade }\end{array}$} & \multirow[t]{4}{*}{1} & Count & 12 & 0 & 6 & 18 \\
\hline & & \% within elastography grade & $66.7 \%$ & $0 \%$ & $33.3 \%$ & $100 \%$ \\
\hline & & $\%$ within MRI grade & $100 \%$ & $0 \%$ & $16.7 \%$ & $30 \%$ \\
\hline & & $\%$ of total & $20 \%$ & $0 \%$ & $10 \%$ & $30 \%$ \\
\hline & \multirow[t]{4}{*}{2} & Count & 0 & 12 & 18 & 30 \\
\hline & & \% within elastography grade & $0 \%$ & $40 \%$ & $60 \%$ & $100 \%$ \\
\hline & & $\%$ within MRI grade & $0 \%$ & $100 \%$ & $50 \%$ & $50 \%$ \\
\hline & & $\%$ of total & $0 \%$ & $20 \%$ & $30 \%$ & $50 \%$ \\
\hline & \multirow[t]{4}{*}{3} & Count & 0 & 0 & 4 & 4 \\
\hline & & \% within elastography grade & $0 \%$ & $0 \%$ & $100 \%$ & $100 \%$ \\
\hline & & \% within MRI grade & $0 \%$ & $0 \%$ & $33.3 \%$ & $20 \%$ \\
\hline & & $\%$ of total & $0 \%$ & $0 \%$ & $20 \%$ & $20 \%$ \\
\hline \multirow{4}{*}{\multicolumn{2}{|c|}{ Total }} & Count & 12 & 12 & 36 & 60 \\
\hline & & \% within elastography grade & $20 \%$ & $20 \%$ & $60 \%$ & $100 \%$ \\
\hline & & \% within MRI grade & $100 \%$ & $100 \%$ & $100 \%$ & $100 \%$ \\
\hline & & $\%$ of total & $20 \%$ & $20 \%$ & $60 \%$ & $100 \%$ \\
\hline
\end{tabular}

Abbreviation: MRI, magnetic resonance imaging. 
Statistical agreement between MRI and elastographic grades was done using kappa statistics. They showed moderate agreement $(\kappa=0.44 ; p<0.001)$.

To test the diagnostic performance of elastography in detecting MRI pathological changes, we considered grade I in both elastography and MRI as normal tendons, while grades II and III were considered pathological. Elastography had $87.5 \%$ sensitivity, $100 \%$ specificity, 100\% PPV, 66.6\% NPV and $90 \%$ accuracy, using our proposed definition of normal and pathological tendons. This finding demonstrates that elastography can be reliably used as a good positive test in symptomatic populations.

\section{Discussion}

Ultrasound elastography provides a noninvasive way to measure tissue mechanical properties in vivo and to assess tissue elasticity that has already shown feasibility in tumor diagnosis as well as to evaluate the mechanical properties of tendons (soft vs. hard). Its application has been expanding rapidly over the past few years with the future potential for early diagnosis, to both guide and monitor therapy.,4,5

The most commonly used method is SEL. The principle of elastography is that tissue compression is done manually by a hand-held US transducer which produces a strain (displacement) within the tissue, and this strain is less in hard tissue than in soft tissue, thus allowing an objective determination of tissue stiffness. ${ }^{6}$

The Achilles tendon was the first area to be investigated using free-hand strain elastography ultrasound. It has provided most of the clinical data available so far in musculoskeletal applications. ${ }^{3}$

El Badry et $\mathrm{al}^{7}$, De Zordo et al, ${ }^{4,8}$ Drakonaki et $\mathrm{al}^{,}{ }^{9}$ and Ooi et $\mathrm{l}^{10}$ agreed that healthy volunteers with asymptomatic Achilles tendons had a predominantly hard texture, whereas Achilles tendons with symptomatic tendinopathy and positive MRI signs of tendinopathy contained softened areas .

SEL alterations found in asymptomatic and sonographically normal tendons are not yet completely understood. It is either due to early (preclinical) changes or false-positive findings, secondary to tissue shifting/nonaxial movement at interfaces between collagen fibers. There are no histopathological or follow-up studies available to prove the above presumptions. ${ }^{3}$ Ooi et $\mathrm{al}^{2}$ stated that their study was the first to indicate that soft tendon characteristic was a significant predictor for the development of symptomatic Achilles tendon injuries. SEL is sensitive to small strain alterations in early Achilles tendon degeneration before pathological changes can be seen with B-mode US or manifest clinically."

In our study, sixty symptomatic patients were examined by ultrasound, SEL and MRI for changes affecting the Achilles tendon.

All 60 patients showed ultrasonographic pathological changes, ranging from only mild insertional irregularities with intact tendon structure, to the full blown picture of Achilles tendinopathy, bursitis and partial tears.
On SEL examination, all 60 tendons showed variable degrees of softening. This result generally agrees with the study of ${ }^{8}$ which showed that symptomatic tendons were found to have areas of softening in $68 \%$ of cases. However, De Sconfienza et al, ${ }^{11}$ found increased stiffness in the abnormal tendons, compared with the nonsymptomatic, which were softer .These results are contradictory, and correlation to histopathology was needed. El Badry et al, ${ }^{7}$ disagreed with De Sconfienza et al, ${ }^{11}$ and stated that "The disparities across various studies are likely attributable to inconsistent techniques, different methodologic approaches or the relatively small study populations in most studies reported. Sconfienza et al, used a different grading system and a lower-frequency-range transducer which may degrade the images acquired and, consequently, could affect their interpretation. So, standardization of SEL techniques should be considered for achieving consistency in its application and allowing more meaningful comparisons between studies."

Klauser et $\mathrm{al}^{12}{ }^{12}$ conducted a study on ten cadavers, the Achilles tendon was assessed histologically after B-mode US and sonoelastographic examination. In their study, both B-mode US and SEL had high specificities, and SEL depicted histologic degeneration (in the form of fiber deviation with fluid accumulation, fatty infiltration; and signs of angiogenesis and neovascularization) more sensitively than B-mode US did (SEL was able to detect histologically confirmed Achilles tendon degeneration in all cases, yet B-mode US could only detect in $85.7 \%$ ). This study supported the elastographic finding of softening in apparently normal tendons by ultrasound alone.

In our study, elastographic detection of softening (blue and green colors)-when using ultrasound as referencehad $100 \%$ sensitivity in detecting abnormalities that were detected by B-mode ultrasound with $16.6 \%$ specificity, positive predictive value of $73 \%$ and negative predictive value of $100 \%$, proving elastography to be a good negative test for tendinopathy .The low specificity can be explained by the work of ${ }^{4}$ who found that $12 \%$ of normal tendons showed mild softening and the work of ${ }^{9}$ who found normal tendons to have areas of softening in $62 \%$ of cases.

In our work, The elastographic blue color representing marked softening appears to correspond more frequently with marked ultrasound changes (focal hypoechoic areas and interstitial tears),While the green color representing mild softening corresponds more frequently with mild or no ultrasonographic changes (thickening, fibrillar pattern interruption, normal/minimal findings).These findings are in common ground with the work of ${ }^{4,13}$ who found that mild softening was not correlated with conventional ultrasound abnormalities, whereas marked softening was found mainly in cases with ultrasound disease, so the authors suggest that only markedly soft areas should be considered as abnormal in Achilles tendon EUS. El Badry et al, ${ }^{7}$ found that SEL revealed alterations in the mechanical properties of the Achilles tendon in 5 tendon segments in whom B-mode US findings were normal. This suggested that SEL is sensitive to small strain alterations in early Achilles tendon degeneration before pathologic changes can be seen with B-mode US. 
Clinically, this may allow early diagnosis and proper management, and can subsequently prevent further progression to more chronic stages where tendons resist treatment.

In our study, correlation of elastography to MRI showed moderate agreement $(\kappa=0.44, p$-value $<0.001)$. This result generally agrees with the study of De Zordo et $a l,{ }^{8}$ in which they found good correlation to MRI $(r=0.8, p$-value $<0.001)$. However, the employed grading system for elastography was different in our study which may explain the statistical difference.

Using MRI as a gold standard, elastography had $87.5 \%$ sensitivity, $100 \%$ specificity, 100\% PPV, $66.6 \%$ NPV, and $90 \%$ accuracy in detecting MR-proved pathological tendons. This finding demonstrates that elastography can be reliably used as a good positive test in symptomatic populations.

We also agreed with Drakonaki et al, ${ }^{9}$ that fat appeared as a mosaic of different colors in the elastogram. This phenomenon occurs mainly from stiffness differences in the boundaries between the fat lobules, fibrous septa, and vessels.

\section{Limitations}

This study has several limitations, first of all the small number of study groups which didn't include normal volunteers. This made it difficult for us to recognize the previously reported overlap of findings between normal and pathological tendons regarding elastographic patterns, and determine which degree of softening can be considered normal.

Also there was another limitation regarding the subjective way of choosing the representative elastogram, which was selected as the one best delineated the tendon boundaries but still could be subject to bias. Also the qualitative nature of image analysis using different grading systems. This has led to variability in the interpretation of the findings, a lower reproducibility and difficulty in comparing the results from different studies .Semi quantitative methods using strain ratios are being developed to partially overcome this limitation.

Technically, the variability of the applied pressure by the hand-held transducer is a known limitation to the free hand technique. The pressure should be moderate, described as the level of pressure that maintains contact with skin and for which the association between pressure and strain is proportional. Very high or low pressure should be avoided, as the elastic properties of tissue become nonlinear.

We didn't have a specific presetting for musculoskeletal elastography in the used ultrasound machine. We didn't have any guide whether a change of the elastographic parameters would influence the imaging quality.

\section{Recommendations}

We recommended that a more systematic and structured approach to the investigation of this new method should be undertaken. First, we advocate standardization of elastographic technique and machine settings for MSK applications, based on the manufacturers' suggestions and consensus between users, standardizing parameters such as the size of the elastogram, the use of adaptors/pads/gel, the scoring systems and so on.

Also, we need to carefully establish the indications for SEL. These would ideally focus on the cohort of patients with symptomatic but non-ultrasound-evident disease, patients at risk or patients at very early stages of disease, to investigate whether SEL is more sensitive than conventional imaging in depicting earlier clinically important changes.

Several technical limitations also need to be overcome to improve the capability of SEL for detecting tendinopathies at an earlier stage. Future longitudinal studies could focus on the potential use of SEL to screen for and perform post treatment follow-up of tendon diseases.

\section{Conclusion}

SEL provides a noninvasive means of estimating tissue stiffness. Using SEL revealed that symptomatic Achilles tendons show variable degrees of softening. The finding of softening corresponds to underlying ultrasonographic changes with high sensitivity, while its low specificity is in need of further research for explanation. The elastographic grades of the tendons showed moderate agreement with MRI grades. Elastography as well proved good specificity and overall diagnostic accuracy in recognizing MR-proven pathological tendons.

We concluded that SEL is a feasible method in diagnosis of Achilles tendon pathologies as complementary to ultrasound examination, and it can be well correlated to MRI. However, further studies are needed mainly for standardization of the technical aspects as well as define the appropriate role of SEL in early detection and thus treatment of Achilles tendinopathy.

\section{Declarations}

Ethics approval and consent to participate:

The current study had been approved by Kasr El-Aini Hospital, Research and Ethical committee. The committee's reference number is not applicable. Verbal consent had been obtained from the patients.

Consent for Publication

Not applicable.

Availability of Data and MaterialThe datasets used and/or analyzed during the current study are available from the corresponding author on reasonable request.

\section{Funding}

None.

\section{Authors' Contributions}

N.M.K. analyzed and interpreted the patient data regarding the sonoelastography of Achilles tendon pathologies. A.M.H. and M.A.A. analyzed and interpreted the data regarding the MRI of Achilles tendon pathologies. N.M.A. revised all the data interpreted by other authors. All authors read and approved the final manuscript.

\section{Conflict of Interest}

None. 


\section{Acknowledgments}

My endless and everlasting thanks to "ALLAH" who enabled me to finish this work appropriately. I would like to express my sincere gratitude and appreciation to Prof. Dr. Nagui Mohamed Abd El Wahab, Professor of Radiodiagnosis, Cairo University, for his generous help, meticulous supervision, valuable advice, and remarks that are inscribed within this work. I wish to thank all my patients without whom this work would never have been completed. Finally, I would like to express my infinite gratitude and my deepest appreciation to my family, all staff members, and to my colleagues, at Radiology Department, for their support.

\section{References}

1 Borga A, El-Essawya S, Roshdy M, Amany E, Ahmed M. Role of ultrasonography in the evaluation of Achilles tendon disorders. Benha Med J 2016;33:54-59

2 Ooi CC, Schneider ME, Malliaras P, et al. Sonoelastography of the Achilles Tendon: prevalence and prognostic value among asymptomatic elite Australian rules football players. Clin J Sport Med 2016;26(4):299-306

3 Drakonaki EE, Allen GM, Wilson DJ. Ultrasound elastography for musculoskeletal applications. Br J Radiol 2012;85(1019) :1435-1445

4 De Zordo T, Fink C, Feuchtner GM, Smekal V, Reindl M, Klauser AS. Real-time sonoelastography findings in healthy Achilles tendons. AJR Am J Roentgenol 2009;193(2):W134-8
5 Li Y, Snedeker JG. Elastography: modality-specific approaches, clinical applications, and research horizons. Skeletal Radiol 2011;40(4):389-397

6 Tan S, Kudaş S, Özcan AS, et al. Real-time sonoelastography of the Achilles tendon: pattern description in healthy subjects and patients with surgically repaired complete ruptures. Skeletal Radiol 2012;41(9):1067-1072

7 El Badry A, Ghieda U, El khouly R, Elreweny E. Evaluation of sonoelastography in Achilles tendon of healthy volunteers and patients with symptomatic Achilles tendon. Egypt Soc Radiol Nucl Med 2018;49:119-127

8 De Zordo T, Chhem R, Smekal V, et al. Real-time sonoelastography: findings in patients with symptomatic Achilles tendons and comparison to healthy volunteers. Ultraschall Med 2010;31(4):394-400

9 Drakonaki EE, Allen GM, Wilson DJ. Real-time ultrasound elastography of the normal Achilles tendon: reproducibility and pattern description. Clin Radiol 2009;64(12):1196-1202

10 Ooi CC, Schneider ME, Malliaras P, Chadwick M, Connell DA. Diagnostic performance of axial-strain sonoelastography in confirming clinically diagnosed Achilles tendinopathy: comparison with B-mode ultrasound and color Doppler imaging. Ultrasound Med Biol 2015;41(1):15-25

11 Sconfienza LM, Silvestri E, Cimmino MA. Sonoelastography in the evaluation of painful Achilles tendon in amateur athletes. Clin Exp Rheumatol 2010;28(3):373-378

12 Klauser AS, Miyamoto H, Tamegger M, et al. Achilles tendon assessed with sonoelastography: histologic agreement. Radiology 2013;267(3):837-842

13 Klauser AS, Faschingbauer R, Jaschke WR. Is sonoelastography of value in assessing tendons? Semin Musculoskelet Radiol 2010;14(3):323-333 